\title{
Engineered cell lines for insulin replacement in diabetes: current status and future prospects
}

\author{
C.B. Newgard ${ }^{1}$, S. C lark' ${ }^{2}$, H . B eltrandelR io ${ }^{1}$, H . E . H ohmeier ${ }^{1}$, C. Q uaade ${ }^{2}$, K. N ormington ${ }^{2}$ \\ ${ }^{1}$ Gifford Laboratories for Diabetes Research and Departments of Biochemistry and Internal Medicine, \\ University of Texas Southwestern Medical Center at Dallas, Dallas, Texas, USA \\ ${ }^{2}$ BetaGene, Inc., Dallas, Texas, USA
}

Summary The recently completed diabetes complications and control trial has highlighted the need for improvement of insulin delivery systems for treatment of insulin-dependent diabetes mellitus. Despite steady improvement in methods for islet and whole pancreas transplantation over the past three decades, the broad-scale applicability of these approaches remains uncertain due in part to the difficulty and expense associated with procurement of functional tissue. To address this concern, we and others have been using the tools of molecular biology to develop cell lines with regulated insulin secretion that might serve as a surrogate for primary islets or pancreas tissue in transplantation therapy. This article seeks to provide a brief summary of the current status of this growing field, with a particular emphasis on progress in producing cell lines with appropriate glucose-stimulated insulin secretion. [Diabetologia (1997) 40: S 42-S 47]

Keywords Insulin, genetic engineering, cell lines, transplantation, molecular biology.
The recently concluded diabetes control and complications trial (DCCT) [1] has emphatically underscored the potential for improvement in current treatment protocols for diabetes mellitus. 'Conventional' insulin therapy, involving 1-2 injections of the hormone per day and intermittent glucose monitoring is clearly not as effective at slowing the progression of the debilitating and costly secondary complications of the disease as 'tight control', in which insulin pumps or multiple injections are combined with frequent glucose monitoring to limit hyperglycaemic excursions. Unfortunately, improvement in glycaemic control through increased insulin dosing requires a new level of compliance and discipline, and places the patient at significantly enhanced risk for dangerous hypoglycaemic episodes. These concerns have

Corresponding author: C. B. Newgard, Ph.D., Gifford Laboratories for Diabetes Research, University of Texas Southwestern Medical Center, 5323 Harry Hines Blvd., Dallas, TX 75235, USA

A bbreviations: DCCT, Diabetes Control and Complications Trial; IDDM, Insulin-dependent diabetes mellitus; GSIS, glucose stimulated insulin secretion. produced a surprising reticence among both physicians and patients towards implementation of tight control therapy. This reaction has served to place a new emphasis on the design of better methods for insulin replacement in insulin-dependent diabetes mellitus (IDDM). A primary consideration in such work is to develop a 'closed loop' system in which the hormone is delivered in response to metabolic demand, thereby eliminating the need for self monitoring by the patient. Three basic approaches to achieving this end have been contemplated, and are being investigated with ever increasing vigour. The first involves development of mechanical devices consisting of a pump or other mechanism for insulin delivery linked to a detector system for blood glucose. The second involves transplantation of insulin-producing cells from human or large mammal donors into IDDM patients. This can be achieved by transplantation of the entire pancreas or isolated pancreatic islets. Because of the difficulty and cost associated with islet isolation and pancreas transplantation, a third approach has emerged, in which the tools of molecular biology are used to fashion insulin-secreting cell lines with fuelmediated insulin secretion responses resembling 
those of normal beta-cells, with the hope that such lines might serve as a surrogate for islets in transplantation therapy. This article seeks to summarize briefly the current status of research on engineered insulinsecreting cell lines and to review the challenges that remain before this technology can be brought forward as a therapeutic alternative.

The islet beta-cell is designed to recognize and integrate a complex array of metabolic and hormonal signals so as to deliver precisely the correct amount of insulin for maintenance of fuel homeostasis. Glucose is the most important stimulator of insulin secretion, not only because of its potent direct effects but also because it is permissive for the stimulatory action of a wide array of other secretagogues. Engineering and maintenance of glucose-stimulated insulin secretion (GSIS) in cell lines that faithfully mimic that of the normal beta-cell is clearly a high priority, but is complicated by our incomplete understanding of the biochemical mechanism(s) of glucose sensing. While there is evidence to suggest that glucose exerts its effect through its own metabolism, resulting in the creation of signals that appear to work through modulation of ion channel activities and influx of extracellular $\mathrm{Ca}^{2+}$, the exact nature of the metabolic coupling factors remains unknown. For a summary of current thinking about these issues, a subject that is beyond the scope of this article, the reader is referred to recent reviews [2-5]. The magnitude of the insulin secretory response appears to be related to the rate of beta-cell glucose metabolism, and both parameters are sharply increased in response to modest increments in extracellular glucose concentrations within the physiological range of 4 to $8 \mathrm{mmol} / \mathrm{l}$. Beta-cells are equipped with the glucose transporter GLUT2 and the glucose phosphorylating enzyme glucokinase which have kinetic properties, particularly a relatively low affinity for glucose, that are ideal for modulation of glucose responsiveness at the concentrations of the sugar encountered in the circulation. Therefore, most of the cellular engineering studies carried out to date have focussed on expression of these gene products in engineered cell lines.

A variety of neuroendocrine cell types contain secretory granules or vesicles that allow acute regulation of peptide hormone release in response to physiological effectors. This property represents an immediate advantage when considering cell types that can serve as a vehicle for insulin replacement. It is also clear, however, that a strategy involving engineering of autologous human endocrine tissue is impractical, because such cells generally exist as small cell populations in relatively inaccessible sites. For this reason, and in an effort to overcome the cost and difficulty associated with isolation of fresh islets, significant effort has been directed at identifying and developing neuroendocrine cell lines that secrete insulin in response to physiological regulators such as glucose.
Table 1. Desired properties for engineered insulin secreting cell lines for insulin replacement in diabetes mellitus

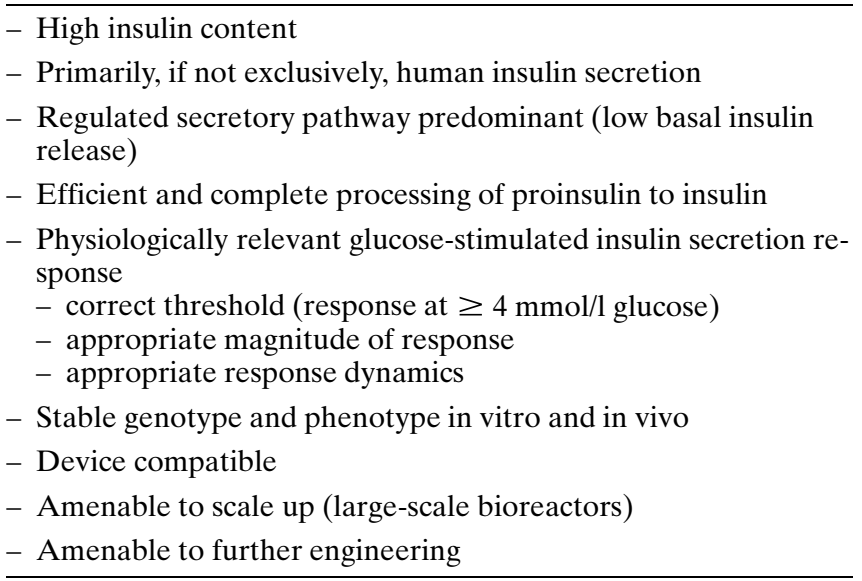

Two basic approaches for achieving this goal have emerged. The first involves modification of pre-existing lines for enhanced function, using the tools of molecular biology. In designing such an approach, it is useful to identify those parameters that are desirable in surrogate beta-cells. Table 1 shows a list of such parameters that we have used as a guide. First, it will be important to ensure that engineered cell lines have high insulin content and secretory capacity. Only in this way can the size of a transplanted graft be restrained to reasonable limits. Second, it will be desirable to engineer the cells so that the insulin produced is predominantly if not exclusively human insulin. Third, it is important that the engineered lines secrete insulin mainly by the so-called 'regulated pathway', as opposed to constitutive release. The regulated pathway encompasses both acute regulation of insulin secretion (i.e. a large increment between the unstimulated and stimulated states) and the complete processing of proinsulin to the mature insulin polypeptide. In islet beta-cells, secretory granules allow the storage of insulin as a depot at the plasma membrane that can be released within seconds of arrival of a fuel-derived or hormonal signal, and also serve as the site of conversion of proinsulin to insulin by virtue of their high concentrations of the relevant convertases PC1 (also known as PC3) and PC2. The presence of secretory granules and retention of proinsulin processing capacity represents a major advantage of insulinoma and other neuroendocrine cell lines relative to cells less specialized for secretion of peptide hormones such as hepatoma cells or fibroblasts (see below). A fourth, and certainly central parameter is to equip the cell with a capacity for glucose sensing. Glucose responsiveness has several components that must be considered, including the appropriate threshold for the response (islet beta-cells only begin to respond to glucose at concentrations in excess of the fasting level of 4-5 mmol/l), rapid response dynamics (beta-cells secrete insulin in response to glucose within minutes of 
its application and turn off insulin secretion nearly as rapidly when glucose is removed) and the correct magnitude of response. Engineering of GSIS has been a central focus of our work and is the major topic that is addressed below.

Should it be possible to generate cell lines with the desired cell biological properties, other issues must also be considered. Continuing with the list in Table 1 , it is imperative that engineered cell lines retain phenotypic and genotypic stability, both in vitro and when implanted into animals or humans. This includes both genes that are inserted or deleted during the course of engineering and key endogenous genes. In the absence of this kind of stability, engineered cells will have no tangible advantage relative to isolated islets, even though procurement of islets is both costly and difficult. Further, it is anticipated that insulin producing cell lines will be transplanted in the context of perm-selective membranes or devices in order to protect the graft from the host immune system, and also to contain the transformed cell lines. The engineered lines must therefore grow and thrive in encapsulation devices, either naturally or as a result of further manipulation of the cells or membrane materials. A major advantage of cell lines relative to islets is that they can be grown in theoretically unlimited quantity at relatively low cost. This advantage can only be realized, however, with the development of methods for scale-up of cell growth from petri dishes to bioreactors. Finally, it will be useful to retain the capacity for further cell engineering, even after development of cells with the aforementioned properties, in case the encapsulation procedures chosen are incapable of fully protecting the implant from the immune system.

Work has been ongoing in our group in all of these areas, with particular focus on engineering of GSIS. Early experiments of this type were carried out in the neuroendocrine cell line AtT-20ins, which is derived from ACTH-secreting corticotrophs of the anterior pituitary. These cells normally do not express the insulin gene, but upon transfection with a plasmid containing the human proinsulin cDNA, are shown to secrete the mature insulin polypeptide [6, 7]. Insulin secretion from AtT-20ins cells can be stimulated by agents such as forskolin or isobutyl methylxanthine (IBMX) [6, 8], but not by glucose [8]. We found that stable transfection of AtT-20ins cells with a plasmid containing the GLUT2 glucose transporter conferred GSIS, albeit with maximal responsiveness occurring at a concentration of approximately $50 \mu \mathrm{mol} / \mathrm{l}$ [9]. Interestingly, transfection of AtT-20ins cells with GLUT1 was without effect, despite the fact that untransfected cells, GLUT1 transfected cells, and GLUT2 transfected cells had very similar rates of 5${ }^{3} \mathrm{H}$ glucose usage [10]. These results suggest that GLUT2 has a role beyond its function as a simple glucose transporter in glucose signalling.
Recently, our attention has turned to the rodent insulinoma cell line RIN1046-38, which is able to respond to glucose at low passage numbers, but loses both glucose sensing and expression of GLUT2 and glucokinase with time in culture $[11,12]$. Stable transfection of these or other glucose unresponsive insulinoma lines such as RINm5F with GLUT2 results in restoration of glucose sensing $[11,13]$. In our studies with intermediate passage number RIN1046-38 cells, restoration of GLUT2 expression was accompanied by a fourfold increase in glucokinase activity [11]. However, the insulin content of these cells is less than $10 \%$ of normal islets and the magnitude of the glucose stimulatory effect is relatively small (2.5fold). In an effort to improve upon these characteristics, we have developed a strategy for the stepwise stable introduction of genes relevant to beta-cell performance, a process that we term 'iterative engineering'. Rodent insulinoma cell lines engineered to contain multiple copies of the human insulin gene exhibit an increase in insulin content of more than 10-fold relative to untransfected cells, and process human proinsulin to mature insulin with high efficiency [14]. Cell lines that are further engineered to express the GLUT2 and glucokinase genes demonstrate stable expression of the three transgenes for the full lifetime of the lines produced to date $(6$ months to 1 year in continuous culture). The multiple engineered lines increase insulin secretion by 6-8-fold in response to glucose, effects that are further potentiated in a fashion similar to that seen in beta-cells by agents that raise cAMP [15]. These responses are clearly larger than observed in unengineered cells, and perifusion studies reveal that insulin secretion is sustained in glucokinase and/or GLUT2 expressing cells more effectively than in unengineered cells or cells that express the human insulin transgene alone [15]. The engineered cell lines exhibit a maximal response to glucose at low concentrations $(0.05-0.25 \mathrm{mmol} / \mathrm{l})$, but can be shifted such that their maximal response occurs at $3-5 \mathrm{mmol} / \mathrm{l}$ glucose by performing secretion studies in the presence of $2 \mathrm{mmol} / \mathrm{l}$ 5-thioglucose, which inhibits low $\mathrm{K}_{\mathrm{m}}$ hexokinase activity and glucose usage by approximately $30 \%$ in these cells [15]. These data suggest that further adjustment of glucose sensing to simulate the response threshold of the beta-cell ( $4-5 \mathrm{mmol} / \mathrm{l})$ should be achievable by stable suppression of low $K_{m}$ hexokinase activity. We are currently investigating several strategies for achieving this goal, including gene 'knock-out' by homologous recombination.

Transplantation of the engineered RIN cell lines into nude rats reveals that stably integrated transgenes are expressed at constant levels in the in vivo environment over the full duration of experiments performed to date (48 days) [14]. Several endogenous genes expressed in normal beta-cells, including rat insulin, amylin, sulfonylurea receptor, glucokinase 
and the transcription factor PDX-1 are stably expressed in the insulinoma lines during these in vivo studies. Endogenous GLUT2 expression, in contrast, is rapidly extinguished (within 10 days of cell implantation). The loss of GLUT2 expression is overcome in engineered cell lines in which GLUT2 expression is provided by a stably transfected transgene [14]. These results suggest that a potential advantage of the iterative engineering approach may be to preserve stability of function and phenotype, particularly in the in vivo setting. These results provide encouragement for upcoming studies on insulin replacement with engineered cells in animal models with chemically induced or spontaneous autoimmune diabetes.

The second approach to generation of new cell lines is to direct expression of a transforming gene, generally simian virus 40 (SV40) T-antigen, to beta-cells of transgenic mice via the insulin promoter/enhancer. Cell lines derived by transgenic expression of T-antigen in beta-cells exhibit variable phenotypes [16-19]. In some cases, these differences have been correlated with expression of glucose transporters and glucose phosphorylating enzymes. Efrat and co-workers [16] have produced a series of such lines known generally as $\beta$ TC. Early lines of this type such as $\beta$ TC- 1 and $\beta$ TC-3 exhibited some GSIS, but were found to be maximally stimulated at concentrations of glucose well below the physiological range. These lines were later found to have high levels of the low $\mathrm{K}_{\mathrm{m}}$ glucose transporter GLUT1 and elevated low $\mathrm{K}_{\mathrm{m}}$ hexokinase activity $[18,19]$. Subsequently, newly isolated lines such as $\beta$ TC- 6 and $\beta$ TC-7 were shown to have a GSIS response that resembled that of the islet in magnitude and concentration dependence [19]. These cells expressed GLUT2 and contained a glucokinase: hexokinase activity ratio similar to that of the normal islet when studied at low passage number, but with time in culture GSIS became maximal at low, subphysiological glucose concentrations. Accompanying this shift in glucose dose-response was a large (approximately 6-fold) increase in hexokinase expression. This enhancement in hexokinase:glucokinase ratio may increase glucose flux at low glucose concentrations, thereby explaining the enhanced sensitivity of the glucose-stimulated insulin secretion response. More recently, soft agar techniques have been used to derive new clonal isolates of $\beta$ TC- 6 cells (such as the clone $\beta$ TC6-F7), which appear to retain differentiated function for longer periods of time in culture [20]. Independently, Miyazaki et al. isolated two classes of clones from transgenic animals expressing an insulin promoter/T-antigen construct [17]. Glucose unresponsive lines such as MIN-7 were found to express GLUT1 rather than GLUT2 as their major glucose transporter isoform, while MIN-6 cells were found to express GLUT2 and to exhibit normal glucose-stimulated insulin secretion. Stable transfection of MIN-6 cells with hexokinase I, but not GLUT1, caused a leftward-shift in the glucose dose-response curve, further underscoring the importance of the low $\mathrm{K}_{\mathrm{m}}$ glucose phosphorylating enzyme in dictating the threshold for glucose responsiveness [21]. A similar change in sensitivity to glucose has recently been demonstrated upon expression of hexokinase I in normal islets, using recombinant adenovirus for efficient delivery of the transgene to islet cells [22]. It remains to be determined whether well-differentiated cell lines produced by transgenic approaches such as MIN-6 or $\beta$ TC6-F7 will retain appropriate levels of expression of glucose transporter and glucose phosphorylating enzyme isoforms when transplanted into diabetic animals for long periods of time. In fact, recent studies show that GLUT2 expression is rapidly extinguished in islets transplanted into diabetic rodents [23], suggesting that stable expression of GLUT2 in this environment may require transfection strategies such as those employed by our group.

Several recent studies on engineering of non-neuroendocrine cell types for insulin delivery deserve mention. Most of this work has focussed on hepatocytes. Delivery of the insulin gene to hepatocytes isolated from a particular patient could be achieved by surgical resection of tissue, ex vivo delivery of the gene of interest with a recombinant viral vector, and reimplantation of the engineered hepatocytes back into the patient. Alternatively, the gene might be delivered to the intact liver by systemic injection or infusion of a recombinant virus. Two recent studies have reported on expression of the insulin gene in hepatocytes in whole animals. Valera et al. [24] used the phosphoenolpyruvate carboxykinase promoter to direct expression of the human insulin gene in transgenic mice. These transgenic mice were normoglycaemic and normoinsulinaemic. Treatment of the animals with the beta-cell cytotoxin streptozotocin resulted in an induction of the human insulin transgene in liver, a tenfold increase in serum levels of human C-peptide, but only a partial correction of hyperglycemia. Kolodka et al. [25] introduced the rat insulin I cDNA into rat liver by performing partial hepatectomy and systemic delivery of a recombinant retrovirus. This treatment resulted in a 3-fold increase in serum immunoreactive insulin and normalization of fasting but not non-fasting blood glucose concentrations. Using an assay specific for free C-peptide Kolodka et al. [25] reported a 10-fold decrease in the serum C-peptide to insulin molar ratio in transgenic animals as compared to normal controls, indicating that a large percentage of the material expressed in liver remains unprocessed. Because hepatocytes lack both regulated secretory granules and the peptidases PC1 (PC3) and PC2 that are responsible for processing proinsulin to insulin, it is perhaps not surprising that the prohormone conversion and regulated insulin release properties of the normal beta-cell are poorly replicated when proinsulin is expressed in the 
liver. Recently, the insulin gene has also been expressed in the HepG2 hepatoma cell line, resulting in the surprising appearance of vacuolar-like structures containing material that reacts with insulin antibodies [26]. Whether these structures represent a storage depot of insulin that can be released in a regulated fashion or an endocytic compartment destined for a degradative pathway remains to be established.

In conclusion, important gains have been made in recent years in identifying and developing cell lines that can perform key functions of the normal islet beta-cell, particularly GSIS. In the near future a major hurdle faced by groups engaged in both the cellular engineering and transgenic approaches will be to establish cell lines that secrete human rather than rodent insulin. While human insulin can be specifically expressed in non-neuroendocrine cell types such as hepatoma cells, it is our view that such cells are not designed for regulated hormone delivery, and that this approach will have limited applicability. Non-islet neuroendocrine cell lines such as AtT-20ins can also be specifically engineered for human insulin production and have the added advantage of the appropriate storage and processing machinery, but it appears that further engineering of these cells to eliminate the secretion of potentially detrimental products such as ACTH will be required [27]. Thus, an immediate focus will be expression of human insulin in rodent insulinoma cells and eliminating expression of the endogenous insulin gene(s). This is necessitated by the lack of a suitable human insulinoma cell line for study, although several groups are currently attempting to produce such lines from human islet tissue. It is clear that the recent intense effort in cell design by genetic engineering will be followed by an equally active period of animal experimentation, wherein the formidable challenges of device design and immunological barriers to transplantation will be addressed. It is in this phase that the therapeutic applicability of this technology will be defined.

A cknowledgements. Studies relevant to this article performed at the Gifford Laboratories for Diabetes Research at the University of Texas Southwestern Medical Center at Dallas were supported by grant R01-DK-46492 from the National Institutes of Health, by a National Institutes of Health/Juvenile Diabetes Foundation International Diabetes Interdisciplinary Research Program, and by a grant from the Redfern Foundation, Midland, Texas.

\section{References}

1. DCCT Group (1993) The effect of intensive treatment of diabetes on the development and progression of long-term complications in insulin-dependent diabetes mellitus. $\mathrm{N}$ Eng J Med 329: 977-986

2. Meglasson MD, Matschinsky FM (1986) Pancreatic islet glucose metabolism and regulation of insulin secretion. Diabetes Metab Rev 2: 163-214
3. MacDonald MJ (1990) Elusive proximal signals of betacells for insulin secretion. Diabetes 39: 1461-1466

4. Liang Y, Matschnisky FM (1994) Mechanisms of action of nonglucose insulin secretagogues. Ann Rev Nutrition 14: 59-81

5. Newgard CB, McGarry JD (1995) Metabolic coupling factors in pancreatic beta-cell signal transduction. Ann Rev Biochem 64: 689-719

6. Moore H-P, Walker MD, Lee F, Kelly RB (1983) Expressing a human proinsulin cDNA in a mouse ACTH-secreting cell. Intracellular storage, proteolytic processing, and secretion on stimulation. Cell 35: 531-538

7. Gross DJ, Halban PA, Kahn RC, Weir GC, Villa-Komaroff L (1989) Partial diversion of a mutant proinsulin (B10 aspartic acid) from the regulated to the constitutive secretory pathway in transfected AtT-20 cells. Proc Natl Acad Sci USA 86: 4107-4111

8. Hughes SD, Quaade C, Milburn JL, Cassidy LE, Newgard CB (1991) Expression of normal and novel glucokinase mRNAs in anterior pituitary and islet cells. $\mathrm{J}$ Biol Chem 266: 4521-4530

9. Hughes SD, Johnson JH, Quaade C, Newgard CB (1992) Engineering of glucose-stimulated insulin secretion and biosynthesis in non-islet cells. Proc Natl Acad Sci USA 9: 688-692

10. Hughes SD, Quaade C, Johnson JH, Ferber S, Newgard CB (1993) Transfection of AtT-20ins cells with GLUT2 but not GLUT1 confers glucose-stimulated insulin secretion: relationship to glucose metabolism. J Biol Chem 268: 1520515212

11. Ferber S, BeltrandelRio H, Johnson JH, Noel R, Becker T, Cassidy LE, Clark S, Hughes SD, Newgard CB (1994) Transfection of rat insulinoma cells with GLUT2 confers both low and high affinity glucose-stimulated insulin release: relationship to glucokinase activity. J Biol Chem 269: $11523-11529$

12. Clark SA, Burnham BL, Chick WL (1990) Modulation of glucose-induced insulin secretion from a rat clonal betacell line. Endocrinology 127: 2779-2788

13. Tiedge M, Hohne M, Lenzen S (1993) Insulin secretion, insulin content, and glucose phosphorylation in RINm5F insulinoma cells after transfection with human GLUT2 glucose transporter cDNA. Biochem J 296: 113-118

14. Clark SA, Quaade C, Constandy H et al. (1997) Novel insulinoma cell lines produced by iterative engineering of GLUT2, glucokinase, and human insulin expression. Diabetes in press

15. Hohmeier HE, BeltrandelRio H, Clark SA, Henkel-Reiger R, Normington K, Newgard CB (1997) Regulation of insulin secretion from novel engineered insulinoma cell lines. Diabetes in press

16. Efrat S, Linde S, Kofod H, Spector D, Delannoy M, Grant S et al. (1988) Beta-cell lines derived from transgenic mice expressing a hybrid insulin gene-oncogene. Proc Natl Acad Sci USA 85: 9037-9041

17. Miyazaki J-I, Araki K, Yamato E et al. (1990) Establishment of a pancreatic $\beta$ cell line that retains glucose-inducible insulin secretion: special reference to expression of glucose transporter isoforms. Endocrinology 127: 126-132

18. Whitesell RR, Powers AC, Regen DM, Abumrad NA (1991) Transport and metabolism of glucose in an insulin-secreting cell line, $\beta$ TC-1. Biochemistry 30: 1156011566

19. Efrat S, Leiser M, Surana M, Tal M, Fusco-Demane D, Fleischer N (1993) Murine insulinoma cell line with normal glucose-regulated insulin secretion. Diabetes 42: 901907 
20. Knaack D, Fiore DM, Surana M et al. (1994) Clonal insulinoma cell line that stably maintains correct glucose responsiveness. Diabetes 43: 1413-1417

21. Ishihara H, Asano T, Tsukuda K et al. (1994) Overexpression of hexokinase I but not GLUT1 glucose transporter alters concentration dependence of glucose-stimulated insulin secretion in pancreatic beta-cell line MIN6. J Biol Chem 269: 3081-3087

22. Becker TC, BeltrandelRio H, Noel RJ, Johnson JH, Newgard CB (1994) Overexpression of hexokinase I in isolated islets of Langerhans via recombinant adenovirus: enhancement of glucose metabolism and insulin secretion at basal but not stimulatory glucose levels. J Biol Chem 269: 21234-21238

23. Thorens B, Wu Y-J, Leahy JL, Weir GC (1992) The loss of GLUT2 expression by glucose-unresponsive $\beta$ cells of $\mathrm{db} /$ $\mathrm{db}$ mice is reversible and is induced by the diabetic environment. J Clin Invest 90: 77-85
24. Valera A, Fillat C, Costa C et al. (1994) Regulated expression of human insulin in the liver of transgenic mice corrects diabetic alterations. FASEB J. 8: 440-447

25. Kolodka TM, Finegold M, Moss L, Woo SLC (1995) Gene therapy for diabetes mellitus by hepatic expression of insulin. Proc Natl Acad Sci USA 92: 3293-3297

26. Simpson AM, Tuch BE, Swan MA, Tu J, Marshall GM (1995) Functional expression of the human insulin gene in a human hepatoma cell line (Hep G2). Gene Therapy 2: 223-231

27. BeltrandelRio H, Schnedl WJ, Ferber S, Newgard CB (1994) Genetic engineering of insulin-secreting cell lines. In: Lanza RP, Chick WL (eds) Pancreatic islet transplantation, vol 1: procurement of pancreatic islets. R. G. Landes. Austin, TX, pp 169-183 